POS $\quad$ PROCEEDINGS

\title{
Reaction Rates for Dense Matter Burning
}

\author{
Mary Beard* and Michael Wiescher \\ University of Notre Dame \\ E-mail: mbeard@nd.edu, wiescher.1@nd.edu
}

\section{Leandro Gasques}

Universidade de Lisboa

E-mail: lgasques@cii.fc.ul.pt

\section{Anatoli Afanasjev}

Mississippi State University

E-mail: aa242@msstate.edu

\section{Dmitry Yakovlev}

Ioffe Physico-Technical Institute

Email: yakeastro.ioffe.rssi.ru

\section{Edward Brown, Kit Yu Lau, and Hendrik Schatz}

National Superconducting Cyclotron Laboratory, Michigan State University

Email: ebrown@pa.msu, lau@nscl.msu.edu, schatz@nscl.msu.edu

\section{W.Raphael Hix}

Oak Ridge National Laboratory

Email: raph@ornl.gov

\section{Sanjib Gupta, Peter Möller, and Andrew Steiner}

Los Alamos National Laboratory

Email: guptasanjib@lanl.gov, moller@lanl.gov, asteiner@lanl.gov

\section{Karl-Ludwig Kratz}

Universität Mainz

Email: klkratz@uni-mainz.de

An interesting question in nuclear astrophysics is the fate of post X-ray burst matter. Once an accreting neutron star has undergone thermonuclear runaway, the ashes of the burst are forced down into the crust of the neutron star. With increasing density a volume element of matter undergoes a number of electron captures and neutron emissions, resulting in low mass neutron rich nuclei. At sufficiently high densities pycnonuclear reactions can set in.

We recently formulated [1] a single phenomenological expression for the calculation of pycnonuclear reaction rates which is not only valid in mixed component plasma, but can also be extended to cover the five stellar burning regimes. Here we use this expression in concert with a dynamic reaction network code to model the neutron star crust composition at high densities.

10th Symposium on Nuclei in the Cosmos

July 27 - August 12008

Mackinac Island, Michigan, USA

\footnotetext{
*Speaker.
} 


\section{Introduction}

Pycnonuclear reactions occur in high density matter when nuclei are frozen in lattice structures. Such matter characterizes the cores of white dwarfs, and the crusts of accreting neutron stars. While pycnonuclear reactions in white dwarf matter are dominated by $12 \mathrm{C}$ and $16 \mathrm{O}$ induced fusion processes, pycnonuclear reactions in neutron star crust matter are dominated by fusion between very neutron rich carbon, oxygen, and neon isotopes at the limits of stability. In a post X-ray burst neutron star binary system ashes from the burst are forced deep into the crust by the weight of freshly accreted matter. With increasing density the remnant abundance distribution is changed by a host of electron capture reactions and neutron emissions, leading to the effective processing of the ashes into very neutron rich nuclei in the carbon to magnesium range. Near nuclear matter densities the nuclei are forced into a lattice, surrounded by a neutralizing degenerate electron gas. Though held in a solid electrostatic lattice, reactions are still possible due to the reduction of Coulomb barrier through electron screening, and the overlap of nuclear wave functions between lattice sites. This type of reaction is highly density dependent, and unlike the more familiar thermonuclear counterpart, is not strictly temperature dependent.

\section{Pycnonuclear Formalism}

Previous work on pycnonuclear reactions has tended to focus more on the simpler case of fusion between identical particles (see for instance [2], [3] and [4]). We have recently extended pycnonuclear formalism for a one component plasma (OCP) to more realistic multicomponent (MC) environments [1]. Our phenomenological approach factors the reaction rate into two terms, one completely temperature independent (known as the $T=0$ pycnonuclear rate), the other dependent on $T$ (known as the thermally enhanced pycnonuclear rate).

Following the results of [4], the $T=0$ rate can be expressed as,

$$
R_{i j}^{\mathrm{pyc}}=10^{46} C_{\mathrm{pyc}} \frac{8 \rho X_{N} x_{i} x_{j} A_{i} A_{j}\langle A\rangle Z_{i}^{2} Z_{j}^{2}}{\left(1+\delta_{i j}\right) A_{c}^{2}} S\left(E_{i j}^{\mathrm{pk}}\right) \widetilde{\lambda}_{i j}^{3-C_{\mathrm{pl}}} \exp \left(-\frac{C_{\mathrm{exp}}}{\left(\widetilde{\lambda}_{i j}\right)^{1 / 2}}\right) \mathrm{cm}^{-3} \mathrm{~s}^{-1}
$$

where $X_{N}$ is the total mass fraction contained in the nucleus $(\approx 1), x_{i}$ and $x_{j}$ are the mass fractions of nucleons $i$ and $j$ respectively, $A_{i}\left(Z_{i}\right), A_{j}\left(Z_{j}\right),\langle A\rangle(\langle Z\rangle)$ are the mass (charge) number of nucleons $i$ and $j$, and the average mass (charge) number. $C_{\text {pyc }}$ is a model dependent parameter, characterizing Coulomb barrier penetration. The density $\rho$ is expressed in $\mathrm{g} \mathrm{cm}^{-3}$ and the astrophysical factor $S\left(E_{i j}^{\mathrm{pk}}\right)$ is in $\mathrm{MeV}$ barn. The reaction energy is $E_{i j}^{p k} \simeq \hbar \omega_{i j}=\alpha_{\omega i j} k_{\mathrm{B}} T_{i j}^{(p)}$. The main parameter dominating the reaction rate is $\widetilde{\lambda}_{i j}$, in the exponent argument. It is given by,

$$
\lambda_{i j}=\frac{A_{i}+A_{j}}{A_{i} A_{j} Z_{i} Z_{j}\left(Z_{i}^{1 / 3}+Z_{j}^{1 / 3}\right)}\left(\frac{\rho X_{N}\langle Z\rangle}{\langle A\rangle 1.3574 \times 10^{11} \mathrm{~g} \mathrm{~cm}^{-3}}\right)^{1 / 3} .
$$

For sufficiently low densities, $\widetilde{\lambda}_{i j}$ is very large, strongly suppressing the Coulomb tunneling. With growing $\rho$ the barrier becomes more transparent and the $T=0$ reaction rate increases. 
Following the familiar thermonuclear reaction rate, thermally enhanced pycnonuclear reactions can be expressed as,

$$
\Delta R_{i j}(T)=\frac{n_{i} n_{j}}{1+\delta_{i j}} \frac{S\left(E_{i j}^{\mathrm{pk}}\right)}{\hbar} r_{\mathrm{B} i j} P F,
$$

where $P$ and $F$ are are parameters containing the exponent describing Coulomb barrier penetrability, $r_{\mathrm{B} i j}$ is a dimensional factor, and $n_{i}\left(n_{j}\right)$ is the number density of $i(j)$.

Combining Eq. (2.1) and Eq. (2.3) gives a full reaction rate expression applicable over a range of temperature and density, from familiar thermonuclear, to exotic pycnonuclear regimes.

\section{Network Simulations of Neutron Star Crust}

Post-process network simulations for the fate of matter in neutron star crusts have recently been performed. The network code is presently developed for including a full network of realistic electron capture (EC) reactions [5] and pycnonuclear fusion. Transition strengths for the EC reactions have been calculated form Gamow-Teller strengths [6] in a quasi-particle random phase approximation. Ground state masses have been obtained from the finite-range droplet model. Neutrinos are handled by calculating the losses for each transition. The EC rates themselves are calculated in real time by using a table of electron capture transition strengths, and a fast analytic phase space approximation [7].

Using a network of $\approx 1500$ isotopes, an accreting neutron star nuclear evolution has been modeled. Realistic X-ray burst abundance ashes [8] were used as the initial starting point (figure $1)$.

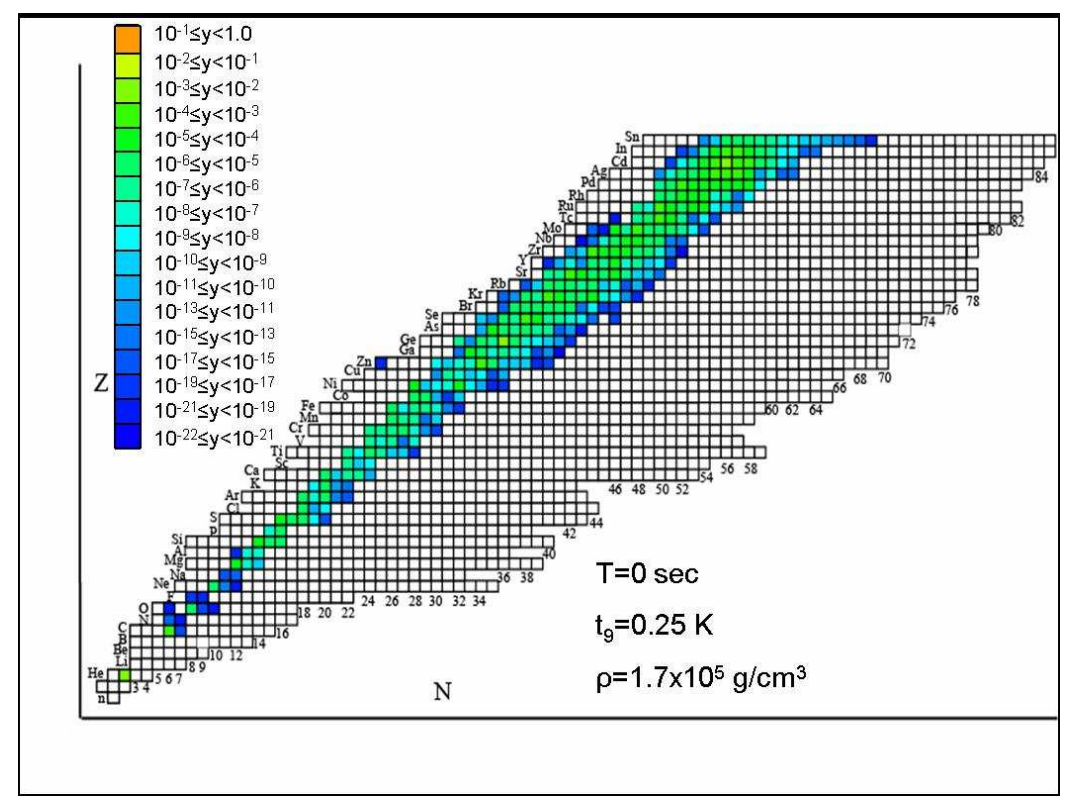

Figure 1: Initial isotopic composition. This composition represents a realistic abundance distribution after an X-ray event [8]. Initial starting conditions were $T_{9}=0.25 \mathrm{~K}, \rho=1.7 \times 10^{5} \mathrm{~g} / \mathrm{cm}^{3}$.

Evolving the simulation with time, we observe the EC rates quickly processing the rapid proton (rp) capture ashes from proton rich material to neutron rich matter. Pycnonuclear reactions could 
be observed taking place in the neutronized matter on a time scale of years. These fusion reactions could represent an important contribution to heating effects in the neutron star crust. As these reactions depend sensitively on the spacing of the nuclei through the $\lambda \hat{\mathrm{I}} \dot{z}$ parameter, we find the lightest nuclei (corresponding to lowest Coulomb barrier) start to burn first, at densities of roughly $1-2 \times 10^{10} \mathrm{~g} / \mathrm{cm}^{3}$ for lightest $C-C$ and $C-O$ systems, and $4-5 \times 10^{10} \mathrm{~g} / \mathrm{cm}^{3}$ for heavier $C-O$, $O-O$ systems and light $C-N e$ systems. At densities in the range of $0.9-1.2 \times 10^{11} \mathrm{~g} / \mathrm{cm}^{3}$ light to midrange $\mathrm{Ne}-\mathrm{Ne}, \mathrm{Ne}-\mathrm{Mg}, \mathrm{Mg}-\mathrm{Mg}$ systems start to undergo pycnonuclear fusion. We also observe the sensitivity of these reactions to mass fraction, $x_{i}$, which can result in heavier, (and even larger $Z$ ), more abundant nuclei burning before lighter nuclei are completely consumed. After a time of $6.1 \times 10^{10} \mathrm{sec}$ (figure 2) EC and subsequent neutron emissions promote the presence of free neutrons in the matter. These neutrons could potentially capture on to the lattice nuclei, in a chain of $(n, \gamma)$ reactions. Work is currently in progress to investigate the possibility of this reaction channel.

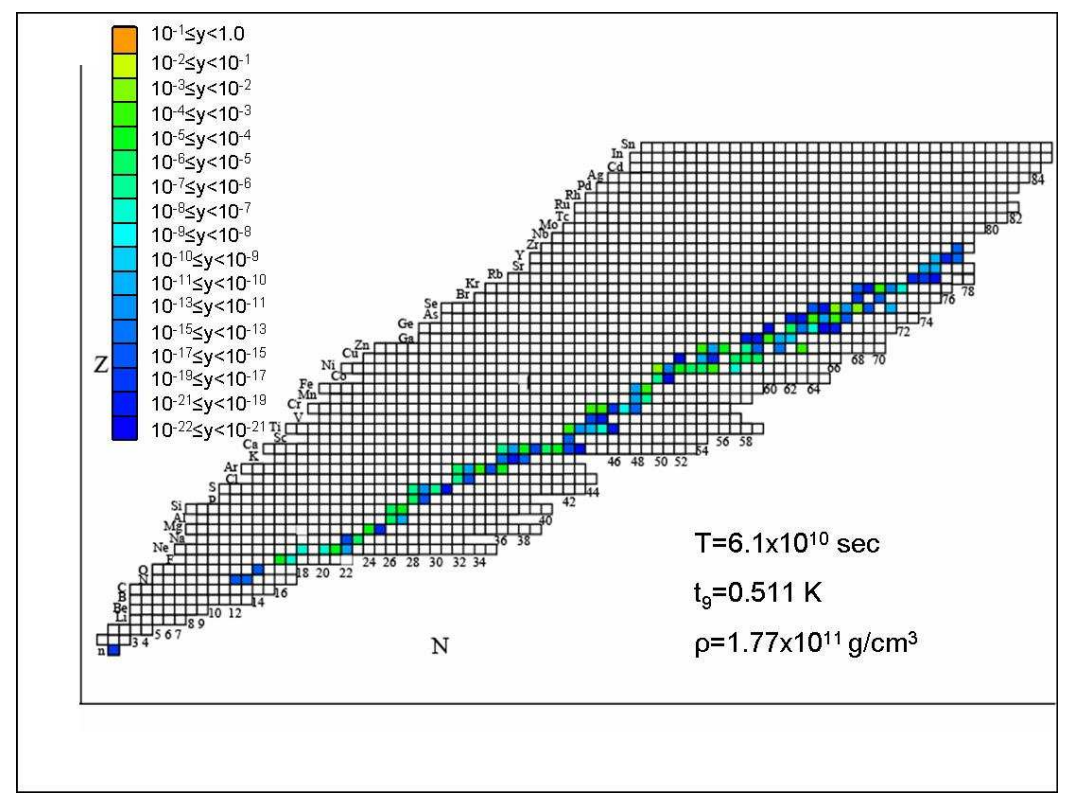

Figure 2: Abundance distribution after $6.1 \times 10^{11}$ seconds. The neutron deficient $\mathrm{rp}$ ashes are effectively processed into neutron rich material.

\section{References}

[1] D. G. Yakovlev, L.R. Gasques, A. V. Afanasjev, M. Beard, M. Wiescher, Phys. Rev. C 74, 035803 (2006).

[2] E. E. Salpeter and H. M. Van Horn, Astrophys. J. 155, 183 (1969).

[3] S. Schramm and S. E. Koonin, Astrophys. J. 365, 296 (1990); erratum: 377, 343 (1991).

[4] L. R. Gasques, A. V. Afanasjev, E. F. Aguilera, M. Beard, L. C. Chamon, P. Ring, M. Wiescher and D. G. Yakovlev, Phys. Rev. C 72, 025806 (2005).

[5] S. Gupta, E. F. Brown, H. Schatz, P. Möller, K.-L. Kratz, Astrophys. J. 662, 2 (2007). 
[6] P. Möller and J. Randrup, Nucl. Phys. A, 514, 1 (1990).

[7] A. Becerril Reyes, S. Gupta, H. Schatz, Electron Capture Rates for Neutron Star Crusts, in proceedings of NIC-IX Conference, POS (NIC-IX) 075.

[8] H. Schatz, A. Aprahamian, J. Görres, M. Wiescher, T. Rauscher, J. Rembges, F.-K. Thielemann, B. Pfeiffer, P. Möller, K.-L. Kratz, H. Herndl, B. A. Brown, H. Rebel, Phys. Reports C 294167 (1998). 